\title{
Efficacy and Safety Balance of Oral and Sublingual Immunotherapy in Food Allergy
}

\section{Valérie Trendelenburg, MSc Kirsten Beyer, MD Katharina Blumchen, $M D^{*}$}

\author{
Address \\ *Department of Pediatric Pneumology and Immunology, \\ Charité-Universitätsmedizin Berlin, \\ Augustenburgerplatz 1, 13353, Berlin, Germany \\ Email: nina.bluemchen@charite.de
}

Published online: 18 May 2014

(C) Springer International Publishing AG 2014

Keywords Oral immunotherapy - Sublingual immunotherapy - Food allergy - Peanut - Hen's egg - Cow's milk • Anaphylaxis · Children · Desensitization - Oral tolerance

\section{Opinion statement}

During recent years, much research has been conducted on oral and sublingual immunotherapy in food allergy. Although efficacy has been shown for this treatment in terms of desensitization, long-term efficacy with regard to tolerance development is not yet proven. More importantly, safety remains a major issue. Although immunotherapy is already performed routinely in some countries, we currently do not recommend this therapeutic approach outside clinical trials. There have been only five randomized placebo-controlled trials but more than 34 reviews on oral immunotherapy in food allergy, indicating that more research is needed. Therefore, the current option for patients with food allergy remains avoidance of the offending food, and because of the constant risk of accidental exposure, patients are advised to use auto-injectable epinephrine in the case of anaphylactic reactions.

\section{Introduction}

Food allergy is a common disease in childhood, with prevalence rates of $4-8 \%$ in industrialized countries $[1,2]$. Some food allergies in early childhood, e.g., cow's milk (CM) and hen's egg (HE) allergy, often resolve over time [3]. On the other hand, peanut or tree nut allergy tends to persist over years [4] and may lead to potentially life-threatening anaphylactic reactions in allergic individuals if the allergen is ingested accidentally [5]. Overall, food allergens are the most frequent elicitors of anaphylaxis in children [6].

Currently, there is no causal treatment option for patients with food allergy; however, trials on specific immu- 
notherapy (IT) using various routes have been performed for many years. The first studies, conducted more than 20 years ago, tried to introduce tolerance to foods via the subcutaneous route $[7,8]$. This treatment option for food allergy has been shown to be effective but unsafe because of frequent, severe adverse reactions. In contrast, the first pilot study investigating epicutaneous IT in children allergic to $\mathrm{CM}$ showed promising results with a good safety profile [9], and larger clinical trials using this approach are on their way (http://clinicaltrials.gov) [10]. Most recently, research was conducted on IT with rectally applied Escherichia coliexpressing recombinant modified food proteins that have a reduced allergenic IgE binding site. Unfortunately, the first pilot trials in humans with peanut allergy did not show completely reduced allergenic reactivity; thus, some patients experienced severe adverse events (AEs) [11]. However, within the past decade, most clinical trials of allergen-specific IT have used the oral (OIT) or sublingual (SLIT) route and have involved different unmodified foods. This review provides an overview of the main efficacy and safety results of OIT and SLIT. Therefore, we reviewed studies of three foods that have been the focus of research in the pediatric population: $\mathrm{CM}, \mathrm{HE}$, and peanut.

\section{Treatment}

\section{Diet}

- Strict allergen avoidance is of prime importance for food allergic patients. However, because of the ubiquitous use of food allergens in the food industry and unregulated precautionary allergen labeling, a complete elimination diet is very difficult. Therefore, patients need professional nutritional recommendations. For patients with peanut and nut allergy, it was shown that professional dietary advice reduces the frequency and severity of accidental reactions [12, 13].

- Food allergy affects children's quality of life to a large extent because of constant uncertainty and fear of allergic reactions [14].

\section{Pharmacologic treatment}

- Patients with food allergy and risk of anaphylactic reactions are advised to carry self-injectable epinephrine. This group of food-allergic patients includes those with a history of anaphylactic reaction; those with systemic reactions to highly potent allergens, such as peanuts/ nuts, with coexisting persistent asthma; those who are far from home or medical facilities; those who have an allergic reaction to small quantities of allergen; and teenagers $[15,16]$.

- Professional training courses on how to manage anaphylaxis and administer emergency self medication may be very helpful for patients and their caregivers $[12,17,18]$. The risk of an accidental reaction was shown to be reduced after professional training programs $[12,13]$.

\section{Oral and sublingual immunotherapy}

\section{Principle}

- The principle of IT is based on administration of very low doses of food protein, with the doses being increased progressively. The objective 
is to achieve first clinical desensitization (i.e., tolerance to a certain amount of the allergen with ongoing therapy) and, later, long-term tolerance (i.e., permanent loss of reactivity that remains after stopping IT).

- Most studies began with an individual or generally defined low allergen dose, followed by a build-up phase with daily administration of the allergen with regular dose increments, e.g., bi-weekly. The dose increments often were performed under medical supervision. Some studies used rush protocols in which the allergen was given in increasing doses several times per day over a short period.

- When a defined target dose (i.e., maintenance dose) is reached, this dose is administered over a defined period (i.e., maintenance phase).

\section{Efficacy}

Maintenance dose

Change in threshold

- OIT efficacy may be defined by different outcome measurements. In this review, we focus on clinical outcome measurements, analyzing the five controlled trials with CM and four with HE performed so far (Table 1). For peanut OIT, we include four uncontrolled studies, as only one controlled trial has been published so far. Concerning the efficacy of OIT, different endpoints may be taken into account, as follows:

With regard to the maintenance dose, it may be distinguished between the amount used and the number of patients tolerating the targeted amounts. The target maintenance dose ranged from 0.5 - $6.6 \mathrm{~g}(12.5-200 \mathrm{~mL}) \mathrm{CM}, 2-6 \mathrm{~g} \mathrm{HE}$ (one third to one small egg), and 0.125 - 4 g peanut protein (1-32 small peanut kernels). Unfortunately, protein levels were not always given by the investigators, so they had to be estimated from the amounts of whole food used. The percentage of patients reaching the OIT maintenance dose ranged from 37-92\% for CM, $0-80 \%$ for HE, and $61-100 \%$ for peanut (Table 1). Taking all of the OIT trials together, the percentage of patients reaching the target maintenance dose (Table 1 , column "Patients reaching maintenance dose") ranged from 0-100\%, making this endpoint a weak parameter for evaluating efficacy. One might assume that as the maintenance dose increases, the number of patients achieving the target dose decreases; yet, this assumption cannot be confirmed when comparing, for example, the studies of Martorell et al. [21] (in which $90 \%$ of patients reached a maintenance dose of $6.6 \mathrm{~g} \mathrm{CM}$ protein) and Longo et al. [19] (in which 37\% of patients reached a maintenance dose of $5 \mathrm{~g}$ ). However, the latter study included older patients with higher CM-specific IgE levels (median $\geq 85 \mathrm{kU} / \mathrm{L}$ ) than those in the study of Martorell et al. (median $10 \mathrm{kU} / \mathrm{L}$ ). So far, only one randomized controlled study has compared two groups with different maintenance doses (1 and $2 \mathrm{~g}$ CM protein) in CM-OIT. Unfortunately, the number of patients who

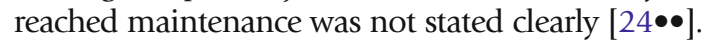

With regard to changes in threshold, the amount of food tolerated before and after OIT or the number of patients with an increase in threshold may be compared. The defined threshold depends on the maximum dose used at food challenge (FC), which varied among studies. For peanut, it ranged from $1-6.6$ g protein (Table 1). Some reports did not give the threshold levels 


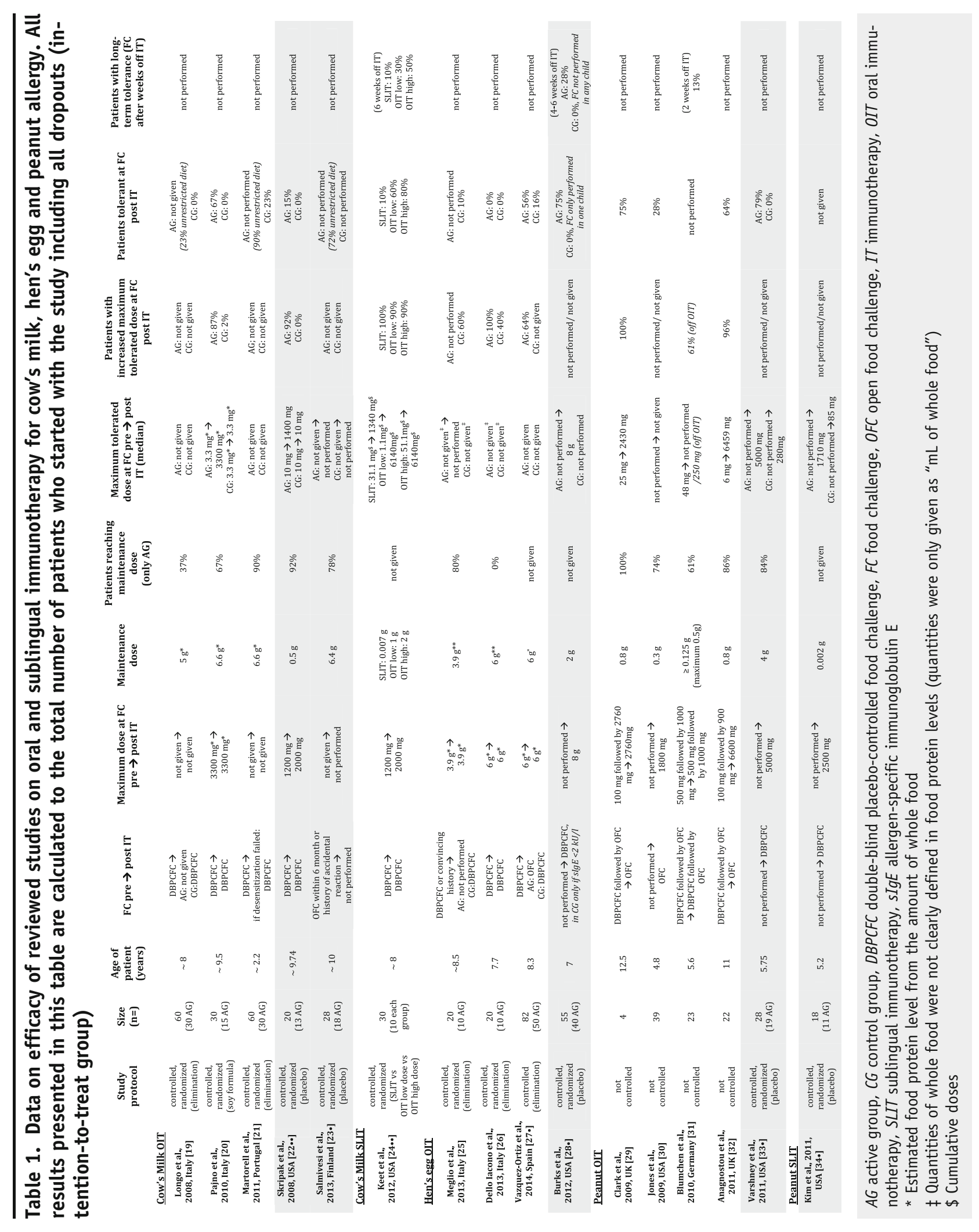


before and after OIT $[19,21,23 \bullet]$, or it was unclear whether the authors referred to the maximum tolerated single or cumulative dose given. Moreover, titration steps, as well as the matrix used at FC, were different [29, 31]. Some studies conducted double-blind placebo-controlled FCs

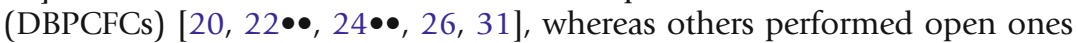
$[27 \bullet, 29,32]$. Sometimes, FCs were performed only post-OIT or only in the control group, or some patients were left out $[19,21,23 \bullet, 25,28 \bullet, 30,33 \bullet$. Therefore, a change in threshold is difficult to compare among the various studies. However, in a few reports, the median maximum tolerated dose at FC before and after OIT was given [Table 1, column "Maximum tolerated dose at FC pre- $\rightarrow$ post-IT (median)"]. For CM, studies reported a median maximum tolerated dose of $1.1-51.1 \mathrm{mg}$ CM protein before and $3300-$ $6140 \mathrm{mg}$ CM protein after OIT $[20,22 \bullet \bullet, 24 \bullet \bullet]$, whereas no threshold in-

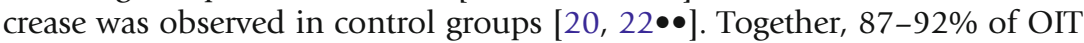
patients and only $0-2 \%$ of control group patients showed an increased tolerated dose at FC (Table 1, column "Patients with increased maximum tolerated dose at FC post-IT"). Only one study with HE-OIT reported the median maximum tolerated dose at FC: $0.45 \mathrm{~mL} \mathrm{HE}$ at baseline, increasing to $20 \mathrm{~mL} \mathrm{HE}$ at final FC in the active group and $0.45 \mathrm{~mL} \mathrm{HE}$ at baseline and at final FC in the control group [26]. One study was not included in our evaluation of this endpoint because its investigators reported doses that evoked symptoms during FC, not the maximum tolerated dose [25]. Three studies on peanut-OIT reported FCs before and after OIT and showed increased maximum tolerated doses: median dose before OIT, range 6-48 mg peanut protein, and after OIT, 250-6,459 mg. In one of these studies, the increase in threshold was much lower, which might be because patients had discontinued OIT 2 weeks before the final FC [31]. After peanut-OIT, 61$100 \%$ of patients increased their threshold dose at FC [29, 31, 32]. In summary, the studies show that $61-100 \%$ of patients receiving OIT can achieve approximately a 5- to $>1,000$-fold increase in their maximum tolerated dose after OIT. Even within one placebo-controlled and two controlled trials, there is clear evidence that an increased threshold can be achieved by patients undergoing OIT. In future studies, a DBPCFC should be performed before and after OIT to evaluate an increased threshold.

Number of patients desensitized

Desensitization in terms of OIT may be defined as patients being tolerant to the maximum dose at FC while undergoing OIT (Table 1, column "Patients tolerant at FC post-IT"). Most OIT studies focused on this outcome to measure efficacy. In CM-OIT, two studies reported desensitization in a large number of patients in the active group (67 and 80\%) [20, 24••], whereas one study reported a smaller number $(15 \%)$ [22・•] (Table 1). This difference might be explained by the fact that the latter study used a much lower maintenance dose than the other two studies (500 vs. 2,000 and 6,600 mg $\mathrm{CM}$ protein). In three controlled trials, none of the patients in the control group developed spontaneous clinical tolerance [19, 20, 22••]. In one study, $23 \%$ of patients developed spontaneous tolerance and did not react at final FC [21]. This study showed the overall highest rate of spontaneous development of tolerance within the control group of all reviewed controlled OIT studies, which might be a result of the inclusion of the overall youngest study population (median age 2.2 years), in whom spontaneous tolerance development still is frequent. In two HE-OIT trials, 56 and $75 \%$ of OIT patients achieved desensitization after treatment $[27 \bullet, 28 \bullet]$. In one study, none of the patients in the active group developed desensitization [26]. In this study, the FC was performed after only 6 months of therapy, compared with $12-$ 22 months of IT in the two other studies. Spontaneous tolerance developed 
Number of patients tolerant

Sublingual immunotherapy in $0-16 \%$ of the control group individuals $[25,26,27 \bullet, 28 \bullet]$. However, in one of these studies, patients in the control group were considered still allergic if egg-specific IgE was $>2 \mathrm{kU} / \mathrm{L}$, and an FC was performed in only one child in the control group [28•]. Peanut-OIT studies reported that $28-75 \%$ of patients were clinically tolerant after treatment in the non-controlled trials [29-32]. The studies differed with regard to maintenance dose, length of maintenance phase, and maximum dose at FC after OIT. In the one published placebo-controlled trial, 79\% of OIT patients became desensitized after 12 months, whereas no one in the placebo group did so [33•]. In summary, OIT studies show that $0-80 \%$ of patients were clinically tolerant after OIT, whereas $0-23 \%$ in the control groups developed natural tolerance. Nonetheless, it has been shown that OIT induces desensitization in many patients. For a more applicable evaluation of the endpoint "desensitization" in prior trials, authors of systemic reviews and meta-analyses of CM-OIT define "desensitization" as the ability of patients to ingest a certain amount of food allergen (e.g., $200 \mathrm{~mL}$ of $\mathrm{CM}$ ) without AEs while on therapy or continued daily ingestion $[35 \bullet \bullet, 36]$. Hence, Yeung et al. $[35 \bullet \bullet]$ showed in a Cochrane analysis that patients receiving CM-OIT had an increased likelihood of achieving desensitization [risk ratio (RR) 6.61] than patients who received an elimination diet alone. Despite this evidence, the authors underscored that the overall quality of evidence was low because of the small number of patients and various methodological differences among studies.

Tolerance in terms of OIT should be defined as patients being tolerant to the maximum dose at FC after discontinuing therapy [Table 1, column "Patients with long-term tolerance (FC after weeks off IT)"]. So far, only a few trials have investigated the induction of tolerance to measure the efficacy of OIT. The first study was a randomized controlled trial comparing OIT with an elimination diet in 45 children allergic to CM or HE [37]. After a median of 21 months, treatment was discontinued for 2 months in OIT children before the final FC was performed. Thirty-six percent of OIT patients and 35\% of patients in the control group were tolerant, indicating no advantage with regard to tolerance induction of OIT compared with an elimination diet [37]. In contrast, Burks et al. [28•] reported that after 22 months of treatment and OIT discontinuation for 4-6 weeks, $28 \%$ of patients in the active group were tolerant to $\mathrm{HE}$ at the final FC, compared with $0 \%$ of those in the placebo group. However, as mentioned earlier, only one of 15 children in the control group was challenged. With regard to tolerance development in peanut-OIT, we conducted an uncontrolled trial with 23 peanut-allergic children [31]. After 9 months of OIT, the patients discontinued OIT for 2 weeks before a DBPCFC was performed. Tolerance was achieved in 13\% of patients. To summarize, more controlled trials are needed to prove OIT efficacy in terms of induction of tolerance. To confirm diagnoses, as well as to determine outcomes, we suggest that a standardized DBPCFC be performed in future studies before, during, and after treatment in all patients in the active and control arms of the study [38, 39].

- Kim et al. [34 $\bullet$ conducted a placebo-controlled trial of SLIT in children with peanut allergy. A DBPCFC performed after 12 months of SLIT or placebo showed that patients in the active group ingested 20 times more peanut protein than those receiving placebo. Unfortunately, no FC was performed at study entry. 
- One study compared SLIT with OIT for CM allergy in 30 patients $[24 \bullet \bullet]$. Ten patients were randomly assigned to a group receiving SLIT alone (target maintenance dose, $7 \mathrm{mg}$ CM protein) or to one of two groups receiving SLIT followed by OIT (target maintenance dose, 1,000 or $2,000 \mathrm{mg}$ CM protein). After at least 48 months of maintenance and IT discontinuation at 6 weeks, $10 \%$ of SLIT patients, $30 \%$ of OIT patients (maintenance dose $1,000 \mathrm{mg}$ ), and $50 \%$ of OIT patients (maintenance dose 2,000 mg) achieved tolerance. Even so, the results suggest a dose-response effect on efficacy due to a higher rate of tolerance induction in patients receiving higher OIT doses; no significant differences between the two OIT groups were found $[24 \bullet \bullet]$.

\section{Safety}

- Despite the promising results of OIT, safety remains a major concern. The most common AEs during OIT are local (e.g., oral pruritus) or gastrointestinal reactions (e.g., abdominal pain, vomiting). More severe AEs affect the respiratory tract (e.g., laryngospasm, wheezing) or are multisystem reactions $[22 \bullet \bullet, 27 \bullet, 28 \bullet, 40]$. However, reporting on safety varies from study to study, which makes evaluation difficult. Moreover, some studies excluded patients with a history of anaphylaxis $[21,22 \bullet \bullet, 30,33 \bullet, 34 \bullet, 41]$ or severe asthma $[22 \bullet \bullet, 28 \bullet]$, whereas other investigators specifically included them $[19,26]$. Furthermore, in some trials, participants were given a prophylactic premedication with antihistamine daily, which would have an impact on reported AEs [19, 25]. Concerning the safety of OIT, different endpoints should be taken into account, as follows:

Some studies reported the number of patients experiencing AEs: nearly all patients (67-100\%) receiving OIT experienced AEs, compared with 0-50\% of patients given placebo (Table 2, column "Patients experiencing adverse events"). Other studies reported the frequency of AEs per dose given and showed that $8-45 \%$ of doses were associated with symptoms $[19,21 \bullet, 25 \bullet \bullet, 28 \bullet, 32 \bullet$, 41], whereas two studies reported symptoms in only $1-3 \%$ of administrated doses in the build-up phase of peanut-OIT [31,33•]. In these two studies, only "clinically relevant" [33•] or objective [31] side effects were assessed as AEs, whereas most other trials also included local, mild reactions (e.g., oral pruritus) and/or subjective side effects (e.g., abdominal pain). In a systematic review and meta-analysis, data from OIT as well as SLIT trials of different food allergies were analyzed and indicated a significant increased risk of local AEs (RR 1.47) and only a non-significant increased risk of systemic AEs with IT (RR 1.08) compared with control groups [42]. Some studies using a combined rush/long-term buildup OIT protocol reported a higher rate of AEs during the initial rush phase than during the build-up or maintenance phase [19, 26, 31, 32, 33•, 40, 43, 44]. In contrast, other studies reported almost equal AE frequency during the different

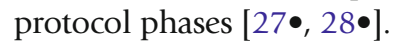


Severity of AEs Several studies assessed the occurrence of severe AEs during OIT, but often

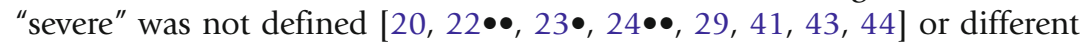

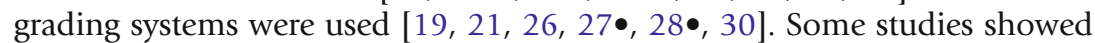
that $0-20 \%$ of patients experienced severe AEs (Table 2 ) during OIT, such as multisystem reactions, including collapse/laryngeal edema or cough/laryngeal edema [20]; grade V reactions (Sampson grading, e.g., respiratory arrest, hypotension, unconsciousness) [27•]; or severe laryngeal symptoms and cough/decreased peak flow [40]. Other studies reported the number of AEs requiring intramuscular (i.m.) epinephrine. As displayed in Table 2 (column "Patients who needed i.m. epinephrine during IT"), 0-31\% of patients received epinephrine during the course of the study in different OIT trials. However, comparing this variable among studies may be precarious. On one hand, differences in the frequency of epinephrine used during OIT might be the result of different country-specific instructions for patients in the treatment of AEs. On the other hand, individual "thresholds" in deciding when to administer epinephrine might differ among individual physicians as well as patients. A systematic review and meta-analysis of the results of four randomized controlled trials of CM-OIT found a higher rate of reactions requiring i.m. epinephrine with OIT (rate ratio 5.8) than in patients in the control group [36]. A Cochrane analysis of CM-OIT showed that for every 11 patients undergoing OIT, one needed i.m. epinephrine

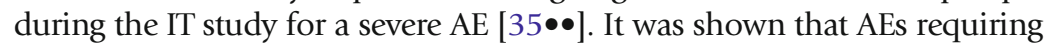
epinephrine occurred not only during dose escalation but also during doses taken at home $[19,22 \bullet \bullet, 30,45]$. In some studies, data regarding epinephrine treatment were not available (Table 2).

Dropout rates Rates of dropout due to AEs ranged from 0 to 36\% (Table 2, column "Dropouts due to adverse events"), which appear higher than in placebo groups $(0-10 \%)[23 \bullet, 28 \bullet]$. Regarding the three foods for which OIT has mostly been investigated so far, rates of dropout due to AEs were comparable: peanut, 0-17\%; CM, 3-20\%; and HE, 0-36\%. In some studies, the occurrence of any severe adverse reaction and/or recurrent AEs despite dose reduction was defined as a reason for OIT discontinuation [19, 26, 27•, 40]. Recurrent AEs leading to discontinuation included respiratory or abdominal

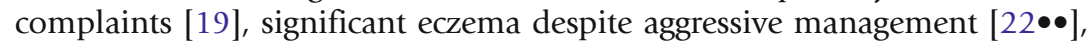
wheezing, and generalized hives, even after IT doses were reduced [28•].

Persistent versus transient AEs

Another possibility for evaluating the safety profile may be the classification of patients into the following groups: (A) patients whose mild AEs resolve over time or are transient (e.g., those who are free of AEs for the last 4 months of OIT); (B) patients with ongoing dose-related AEs over the whole OIT period or with persistent AEs (e.g., ongoing reactions for the last 4 months of OIT); and (C) patients who discontinued OIT because of frequent AEs not improved by reduction and readaptation of OIT doses. This classification was proposed by Vázquez-Ortiz et al. [27•], who evaluated the safety of HE-OIT. When they recorded safety data of 50 patients, $48 \%$ corresponded to the subgroup with transient AEs (A), 34\% to patients with ongoing AEs (B), and $18 \%$ to patients who dropped out because of AEs (C). In comparing these results with baseline parameters of the subgroups, the authors showed that baseline egg-specific IgE is a helpful predictor of the probability of ongoing AEs during OIT. If baseline ovomucoid-specific IgE was $>8.9 \mathrm{kU} / \mathrm{L}$, there was a $95 \%$ probability that patients belonged to group B or C. Similar results were shown for CM allergy [46].

Number of patients "difficult to treat."

Persistent AEs during the induction or build-up phase of OIT may lead to an increased number of dose reductions, which may prolong the treatment 


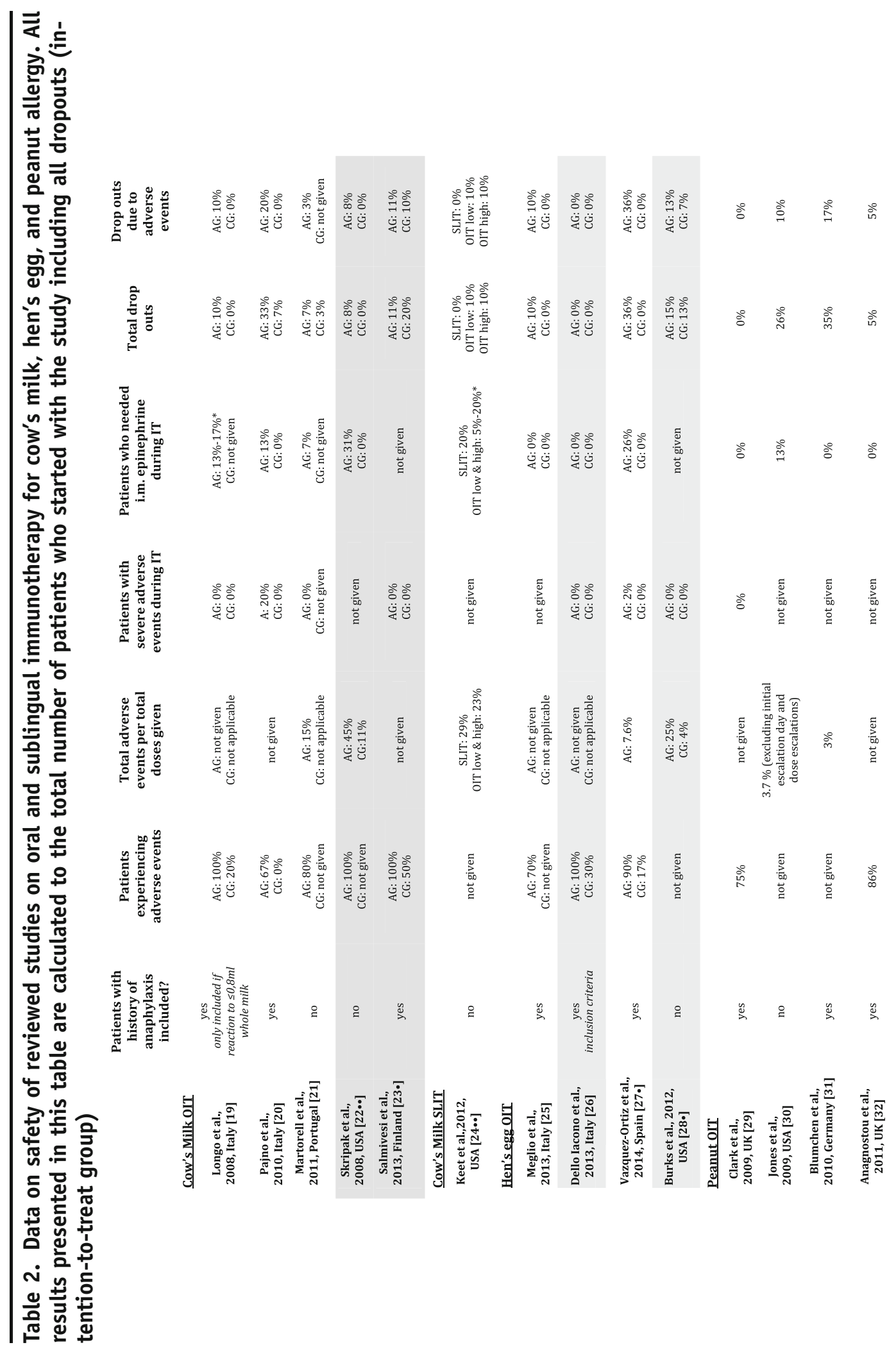




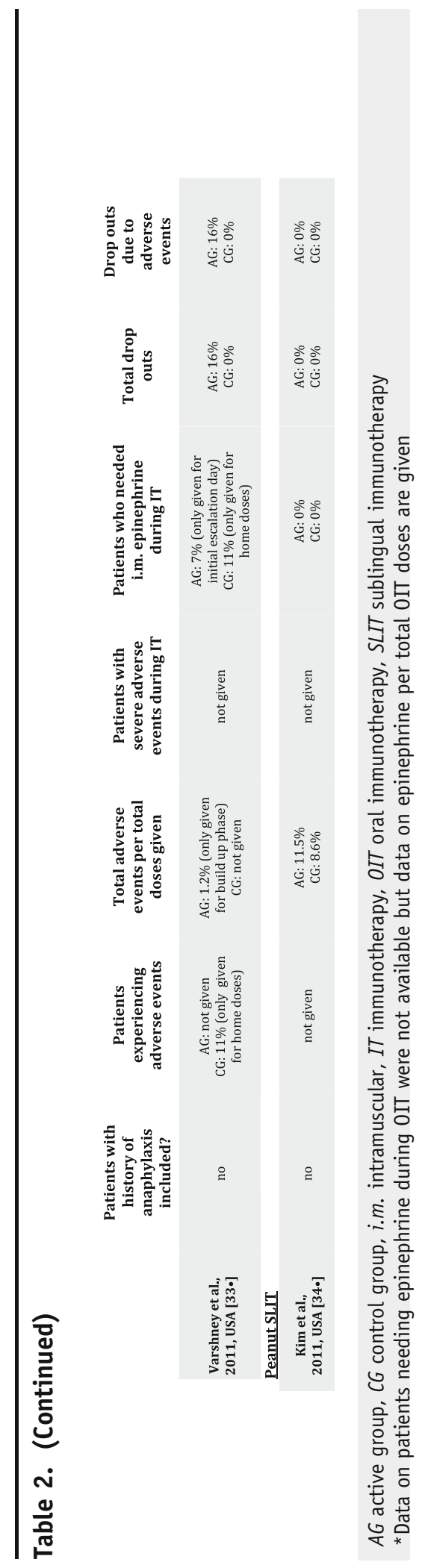


period until reaching a maintenance dose [32]. Anagnostou et al. [32] defined "difficult to treat" as the number of dose reductions during peanut-OIT and found baseline peanut-specific IgE to be a possible predictor of patients requiring dose reduction during OIT. None of the patients $(8 / 8)$ with baseline peanut-specific IgE $<27.3 \mathrm{kU} / \mathrm{L}$ needed a dose adjustment, whereas five of 13 patients with baseline peanut-specific $\operatorname{IgE} \geq 27.3 \mathrm{kU} / \mathrm{L}$ needed a dose reduction.

Augmentation factors Staden et al. [37] first reported an increased risk of AEs caused by "augmentation factors," which seemed to decrease threshold doses in CM- and HE-OIT. Subsequent research on peanut-OIT identified such co-factors as well $[32,47]$. Several augmentation factors have been described, such as infections, poorly controlled asthma, co-exposure to other allergens, exercise, tiredness, anxiety, and menstruation [32, 37, 47]. Recently, Vázquez-Ortiz et al. [46] reported a newly found co-factor: post-intake "lying down" was associated with respiratory AEs. The authors assumed that silent gastroesophageal reflux might be the underlying mechanism.

- It was hypothesized that SLIT has a better safety profile than OIT, as also

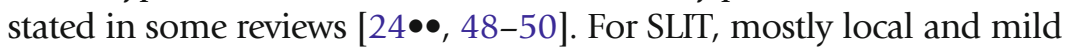

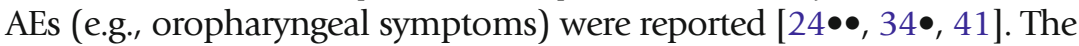
only prospective study directly comparing SLIT with OIT for CM allergy showed similar AE rates during therapy in both groups. However, multisystem as well as lower and upper respiratory tract reactions were more

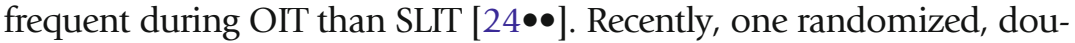
ble-blind, placebo-controlled multicenter trial of peanut-SLIT reported the results of 40 peanut-allergic patients (age range 12-37 years) randomly assigned 1:1 to peanut-SLIT or placebo [41]. After 44 weeks of SLIT, $40.1 \%$ of SLIT doses were associated with AEs, compared with only $0.6 \%$ of placebo doses. Excluding oropharyngeal symptoms, which accounted for most AEs during SLIT, only 5.3\% of SLIT doses were associated with symptoms. Therefore, the authors concluded that SLIT might be a relatively safe option for desensitization in peanut allergy. However, although to a lesser extent, even in trials with SLIT, up to $20 \%$

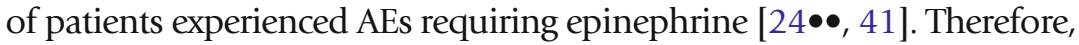
more controlled trials comparing SLIT and OIT head-to-head are needed to evaluate their safety profile.

\section{Balance}

- Two meta-analyses proved OIT efficacy in IgE-mediated CM allergy, leading to desensitization in most patients $[35 \bullet \bullet, 36]$. Nonetheless, some authors stated an overall low quality of evidence because of the small number of included randomized controlled trials, with a small number of patients, and methodological limitations due to signifi- 


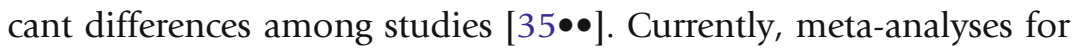
HE- and peanut-OIT are still absent because of the small number of randomized controlled studies [51]. Thus, larger randomized controlled trials with high methodological quality, such as DBPCFCs before and after OIT in the active and control groups, are needed. Studies directly comparing different protocols, such as different maintenance doses, might help establish standardized protocols for future OIT trials. Although OIT seems to lead to desensitization, which protects patients against accidental reactions during ongoing therapy, so far there is no consistent evidence for the induction of long-term tolerance after discontinuation of OIT.

- So far, meta-analyses have reported only poor results regarding the safety

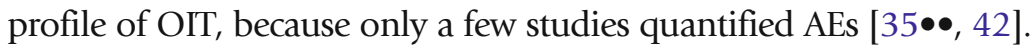

There is great variation in reporting AEs and the use of severity grading systems. For a comparable safety profile among OIT studies, the outcome measurements proposed earlier should be used. Nevertheless, OIT seems to lead to a higher risk of AEs than an elimination diet. Approximately $67-100 \%$ of patients in the active groups, but only $0-50 \%$ in the control groups, experienced AEs. Regarding all studies reviewed in this article in which data were given (Table 2), a total of 35-39 of 364 patients (10$11 \%$ ) undergoing OIT or SLIT received epinephrine, compared with one of 175 patients $(0.6 \%)$ in the control groups. This should be taken into account, especially in younger patients with CM or HE allergy and a good chance of developing spontaneous tolerance.

- Furthermore, the long-term safety of OIT has not been investigated thoroughly. A few cases of eosinophilic esophagitis were reported in OIT trials $[40,46,52]$, but it is still uncertain whether these were related to the treatment.

- Concerning safety, predictors are needed to identify patients at high risk for frequent and severe AEs during treatment. OIT may be the therapy of choice only for some food-allergic patients. This group of patients would benefit from OIT, with no or only mild, transient AEs [27•]. On the other hand, there might be a group of patients, i.e., non-responders, in whom desensitization is not possible because of persistent, severe AEs. For this group of "high-risk" patients, other protocols or different forms of IT are needed. Future attempts to improve the safety of OIT include combination treatment with anti-IgE $[53,54]$, the use of heat-denatured proteins [55], and an alternate route of administration, such as epicutaneous IT [9].

- Although this review aims to compare studies, it should be taken into account that the baseline characteristics of each trial varied with regard to, for example, the number of patients included in each trial (range, $n=4-82$ ); age of patients (median age range, 2.2-12.5 years); inclusion of high-risk patients (yes/no); allergen-specific IgE of patients at baseline (e.g., median peanut-specific IgE 30-96 kU/L); and OIT protocol, such as the target maintenance dose (e.g., 1254,000 mg peanut protein), performance of an FC before and after treatment, and maximum dose given during FC (e.g. 1,000- 
6,600 mg peanut protein), making comparison among the studies very challenging.

- SLIT appears to be less efficient than OIT; for example, patients seem to reach a lower increase in threshold and fewer patients seem to become desensitized. In a placebo-controlled study of peanut-SLIT, 14 of 20 SLIT patients (70\%) showed a tenfold increase in their maximum tolerated dose at FC after SLIT, compared with three of 20 patients (15\%) receiving placebo, whereas none of the patients in the active group and three in the control group could pass the FC after 44 weeks of SLIT [41]. However, SLIT appears to have a better safety

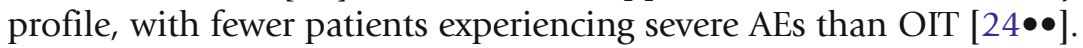
Therefore, SLIT might be an option for the group of high-risk patients with persistent, severe AEs on OIT; it may lead to an increased threshold in many patients, which might protect them against reactions after accidental exposure, and it has a better safety profile than OIT. However, there have not been enough studies directly comparing efficacy and safety between OIT and SLIT.

\section{Compliance with Ethics Guidelines}

\section{Conflict of Interest}

Kirsten Beyer has received consulting or speaker's fees from Danone, MedaPharma, ALK, Novartis, Unilever, Allergopharma, MedUpdate, HAL, Hipp, Mead Johnson, ECARF Institute, and Infectopharm and funding from the European Union, German Research Foundation, ThermoFisher, Danone, DST, FAAN, and the Foundation for the Treatment of Peanut Allergy.

Katharina Blumchen has received speaker's fees from MedaPharma and Novartis and funding from Nutricia Research Foundation, Foundation for the Treatment of Peanut Allergy, and Berliner Sparkassenstiftung Medizin.

Human and Animal Rights and Informed Consent

This article does not contain any studies with human or animal subjects performed by any of the authors.

\section{References and Recommended Readin}

Papers of particular interest, published recently, have been highlighted as:

- Of importance

$\bullet \quad$ Of major importance

1. Rona RJ, Keil T, Summers C, et al. The prevalence of food allergy: a meta-analysis. J Allergy Clin Immunol. 2007;120(3):638-46.

2. Zuidmeer L, Goldhahn K, Rona RJ, et al. The prevalence of plant food allergies: a systematic review. J

Allergy Clin Immunol. 2008;121(5):1210-8. e1214.

3. Host A, Halken S. A prospective study of cow milk allergy in Danish infants during the first 3 years of life. Clinical course in relation to clinical and im- 
munological type of hypersensitivity reaction. Allergy. 1990;45(8):587-96.

4. Skolnick HS, Conover-Walker MK, Koerner CB, et al The natural history of peanut allergy. J Allergy Clin Immunol. 2001;107(2):367-74.

5. Pumphrey RS. Lessons for management of anaphylaxis from a study of fatal reactions. Clin Exp Allergy. 2000;30(8):1144-50.

6. Hompes S, Kohli A, Nemat K, et al. Provoking allergens and treatment of anaphylaxis in children and adolescents-data from the anaphylaxis registry of German-speaking countries. Pediatr Allergy Immunol. 2011;22(6):568-74.

7. Oppenheimer JJ, Nelson HS, Bock SA, et al. Treatment of peanut allergy with rush immunotherapy. J Allergy Clin Immunol. 1992;90(2):25662.

8. Nelson HS, Lahr J, Rule R, et al. Treatment of anaphylactic sensitivity to peanuts by immunotherapy with injections of aqueous peanut extract. J Allergy Clin Immunol. 1997;99(6 Pt 1):744-51.

9. Dupont C, Kalach N, Soulaines P, et al. Cow's milk epicutaneous immunotherapy in children: a pilot trial of safety, acceptability, and impact on allergic reactivity. J Allergy Clin Immunol. 2010;125(5):1165-7.

10. Otsu K, Fleischer DM. Therapeutics in food allergy: the current state of the art. Curr Allergy Asthma Rep. 2012;12(1):48-54.

11. Wood RA, Sicherer SH, Burks AW, et al. A phase 1 study of heat/phenol-killed, E. coli-encapsulated, recombinant modified peanut proteins Ara h 1, Ara h 2, and Ara h 3 (EMP-123) for the treatment of peanut allergy. Allergy. 2013;68(6):803-8.

12. Ewan PW, Clark AT. Efficacy of a management plan based on severity assessment in longitudinal and case-controlled studies of 747 children with nut allergy: proposal for good practice. Clin Exp Allergy. 2005;35(6):751-6.

13. Clark AT, Ewan PW. Good prognosis, clinical features, and circumstances of peanut and tree nut reactions in children treated by a specialist allergy center. J Allergy Clin Immunol. 2008;122(2):286-9.

14. Avery NJ, King RM, Knight S, et al. Assessment of quality of life in children with peanut allergy. Pediatr Allergy Immunol. 2003;14(5):378-82.

15. Niggemann B, Beyer K. Adrenaline autoinjectors in food allergy: in for a cent, in for a euro? Pediatr Allergy Immunol. 2012;23(6):506-8.

16. Simons FE, Ardusso LR, Bilo MB, et al. World Allergy Organization anaphylaxis guidelines: summary. J Allergy Clin Immunol. 2011;127(3):587-93. e581-522.

17. Muraro A, Roberts G, Clark A, et al. The management of anaphylaxis in childhood: position paper of the European academy of allergology and clinical immunology. Allergy. 2007;62(8):857-71.
18. Ring J, Beyer K, Dorsch A, et al. Anaphylaxieschulung - ein neues Behandlungsprogramm zur tertiären Krankheitsprävention nach Anaphylaxie. Allergo J. 2012;21(2):96-102.

19. Longo G, Barbi E, Berti I, et al. Specific oral tolerance induction in children with very severe cow's milkinduced reactions. J Allergy Clin Immunol. 2008;121(2):343-7.

20. Pajno GB, Caminiti L, Ruggeri $\mathrm{P}$, et al. Oral immunotherapy for cow's milk allergy with a weekly updosing regimen: a randomized single-blind controlled study. Ann Allergy Asthma Immunol. 2010;105(5):376-81.

21. Martorell A, De la Hoz B, Ibáñez MD, et al. Oral desensitization as a useful treatment in 2-year-old children with cow's milk allergy. Clin Exp Allergy. 2011;41(9):1297-304.

22.• Skripak JM, Nash SD, Rowley H, et al. A randomized, double-blind, placebo-controlled study of milk oral immunotherapy for cow's milk allergy. J Allergy Clin Immunol. 2008;122(6):1154-60.

Like the study by Salmivesi et al. [22], this is a randomized, placebo-controlled, double-blind study of OIT in CM-allergic children. To evaluate the efficacy of the treatment, a DBPCFC was performed before and after OIT in the active and control groups

23. Salmivesi S, Korppi M, Mäkelä MJ, et al. Milk oral immunotherapy is effective in school-aged children. Acta Paediatr. 2013;102(2):172-6.

This is one of two randomized, placebo-controlled, doubleblind trials of OIT in CM-allergic children. Unfortuntely, the efficacy of OIT was not assesed by FC. However, most children in the active group could consume daily $\mathrm{CM}$ or $\mathrm{CM}$ products, which was assesed by phone interviews 12 months after OIT.

24.• Keet CA, Frischmeyer-Guerrerio PA, Thyagarajan A, et al. The safety and efficacy of sublingual and oral immunotherapy for milk allergy. J Allergy Clin Immunol. 2012; 129(2): 448-455.e445.

This study, the only one directly comparing SLIT and OIT in $\mathrm{CM}$ allergy, showed OIT to be more efficacious but also associated with more systemic AEs than SLIT.

25. Meglio P, Giampietro PG, Carello R, et al. Oral food desensitization in children with IgE-mediated hen's egg allergy: a new protocol with raw hen's egg. Pediatr Allergy Immunol. 2013;24(1):75-83.

26. Dello Iacono I, Tripodi S, Calvani M, et al. Specific oral tolerance induction with raw hen's egg in children with very severe egg allergy: a randomized controlled trial. Pediatr Allergy Immunol. 2013;24(1):66-74.

27. Vázquez-Ortiz M, Alvaro M, Piquer M, et al. Baseline specific IgE levels are useful to predict safety of oral immunotherapy in egg-allergic children. Clin Exp Allergy. 2014;44(1):130-41.

This article first proposes predictors of dose-related reactions in OIT. Egg-specific IgE was found to identify patients at higher risk for AEs during OIT. 
28. Burks AW, Jones SM, Wood RA, et al. Oral Immunotherapy for treatment of egg allergy in children. $\mathrm{N}$ Engl J Med. 2012;367(3):233-43.

This is the only placebo-controlled, double-blind study of OIT in children allergic to HE. After 22 months of OIT, treatment was discontinued for 4-6 weeks to evaluate long-term tolerance. Unfortuntely, most patients in the placebo group were not challenged, but peanut allergy was determined through elevated peanut-specific IgE levels.

29. Clark AT, Islam S, King Y, et al. Successful oral tolerance induction in severe peanut allergy. Allergy. 2009;64(8):1218-20.

30. Jones SM, Pons L, Roberts JL, et al. Clinical efficacy and immune regulation with peanut oral immunotherapy. J Allergy Clin Immunol. 2009;124(2):292300. e297.

31. Blumchen $\mathrm{K}$, Ulbricht $\mathrm{H}$, Staden $\mathrm{U}$, et al. Oral peanut immunotherapy in children with peanut anaphylaxis. J Allergy Clin Immunol. 2010;126(1):83-91. e81.

32. Anagnostou K, Clark A, King Y, et al. Efficacy and safety of high-dose peanut oral immunotherapy with factors predicting outcome. Clin Exp Allergy. 2011;41(9):1273-81.

33. Varshney P, Jones SM, Scurlock AM, et al. A randomized controlled study of peanut oral immunotherapy: clinical desensitization and modulation of the allergic response. J Allergy Clin Immunol. 2011;127(3):654-60.

This is the only placebo-controlled, double-blind OIT study in peanut-allergic children. After 1 year of OIT, $79 \%$ of the active group and $0 \%$ of the control group showed no clinical reaction during FC. However, an FC was not performed before OIT.

34. $\quad$ Kim EH, Bird JA, Kulis M, et al. Sublingual immunotherapy for peanut allergy: clinical and immunologic evidence of desensitization. J Allergy Clin Immunol. 2011; 127(3): 640-646.e641.

In this study, 18 peanut-allergic children received either peanut-SLIT or placebo over a 12-month period. SLIT patients could ingest 20 times more peanut protein at final challenge than control group patients. However, no FC was performed before OIT.

35.• Yeung JP, Kloda LA, McDevitt J, et al. Oral immunotherapy for milk allergy. Cochrane Database Syst Rev. 2012;11, CD009542.

This Cochrane review of OIT for milk allergy included five randomized controlled trials. Efficacy in terms of desensitization was shown, whereas the overall quality of evidence was low because of small numbers of patients.

36. Brożek JL, Terracciano L, Hsu J, et al. Oral immunotherapy for IgE-mediated cow's milk allergy: a systematic review and meta-analysis. Clin Exp Allergy. 2012;42(3):363-74.

37. Staden U, Rolinck-Werninghaus C, Brewe F, et al. Specific oral tolerance induction in food allergy in children: efficacy and clinical patterns of reaction. Allergy. 2007;62(11):1261-9.
38. Plaut M, Sawyer RT, Fenton MJ. Summary of the 2008 National Institute of Allergy and Infectious Diseases-US Food and Drug Administration Workshop on Food Allergy Clinical Trial Design. J Allergy Clin Immunol. 2009;124(4):671-8. e671.

39. European Medicines Agency committee for medical products for human use (CHMP). Guideline on the clinical development of products for specific immunotherapy for the treatment of allergic diseases/Doc. Ref. CHMP/EWP/18504/2006. http://www.ema.europa.eu/docs/en_GB/ document_library/Scientific_guideline/2009/09/ WC500003605.pdf. Accessed 15 Jan 2014.

40. Hofmann AM, Scurlock AM, Jones SM, et al. Safety of a peanut oral immunotherapy protocol in children with peanut allergy. J Allergy Clin Immunol. 2009;124(2):286-91. e286.

41. Fleischer DM, Burks AW, Vickery BP, et al. Sublingual immunotherapy for peanut allergy: a randomized, double-blind, placebo-controlled multicenter trial. J Allergy Clin Immunol. 2013;131(1):119-27. e117.

42. Nurmatov U, Devereux G, Worth A, et al. Effectiveness and safety of orally administered immunotherapy for food allergies: a systematic review and metaanalysis. Br J Nutr. 2013;111(1):12-22.

43. Buchanan AD, Green TD, Jones SM, et al. Egg oral immunotherapy in nonanaphylactic children with egg allergy. J Allergy Clin Immunol. 2007;119 (1):199-205.

44. Vickery BP, Pons L, Kulis M, et al. Individualized IgEbased dosing of egg oral immunotherapy and the development of tolerance. Ann Allergy Asthma Immunol. 2010;105(6):444-50.

45. Barbi E, Longo G, Berti I, et al. Adverse effects during specific oral tolerance induction: in home phase. Allergol Immunopathol (Madr). 2012;40(1):41-50.

46. Vázquez-Ortiz M, Álvaro-Lozano M, Alsina L, et al. Safety and predictors of adverse events during oral immunotherapy for milk allergy: severity of reaction at oral challenge, specific IgE and prick test. Clin Exp Allergy. 2013;43(1):92-102.

47. Varshney P, Steele PH, Vickery BP, et al. Adverse reactions during peanut oral immunotherapy home dosing. J Allergy Clin Immunol. 2009;124(6):1351-2.

48. De Boissieu D, Dupont C. Sublingual immunotherapy for cow's milk protein allergy: a preliminary report. Allergy. 2006;61(10):1238-9.

49. Kostadinova AI, Willemsen LE, Knippels LM, et al. Immunotherapy - risk/benefit in food allergy. Pediatr Allergy Immunol. 2013;24(7):633-44.

50. Narisety SD, Keet CA. Sublingual vs oral immunotherapy for food allergy: identifying the right approach. Drugs. 2012;72(15):1977-89.

51. Nurmatov U, Venderbosch I, Devereux G, et al. Allergen-specific oral immunotherapy for peanut allergy. Cochrane Database Syst Rev. 2012;9, CD009014. 
52. Ridolo E, De Angelis GL, Dall'Aglio P. Eosinophilic esophagitis after specific oral tolerance induction for egg protein. Ann Allergy Asthma Immunol.

2011;106(1):73-4.

53. Nadeau KC, Schneider LC, Hoyte L, et al. Rapid oral desensitization in combination with omalizumab therapy in patients with cow's milk allergy. J Allergy Clin Immunol. 2011;127(6):1622-4.
54. Schneider LC, Rachid R, LeBovidge J, et al. A pilot study of omalizumab to facilitate rapid oral desensitization in high-risk peanut-allergic patients. J Allergy Clin Immunol. 2013;132(6):1368-74.

55. Leonard SA, Sampson HA, Sicherer SH, et al. Dietary baked egg accelerates resolution of egg allergy in children. J Allergy Clin Immunol. 2012;130(2):473-80. e471. 